\title{
Design of Multi-rotor Control System Basing on Coaxial Propellers
}

\author{
Yunqi Zhang, Yong $\mathrm{Xu}^{*}$ and Hangzhou Zhao \\ 5 South Zhongguancun Street, Haidian District, Beijing, China \\ ${ }^{*}$ Corresponding author
}

\begin{abstract}
A kind of UAV with coaxial propellers and multi-rotor is presented and the dynamics model of this UAV is established in this paper, then the control system of the UAV is designed on the basis of PID control algorithm, which includes altitude and attitude control module. Simulation results show that the control system can realize effective altitude control and attitude control of the multi-rotor aircraft.
\end{abstract}

Keywords-multi-rotor aircraft; coaxial propellers; control system; PID control

\section{INTRODUCTION}

Currently, the prospect of multi-rotor UAV is very extensive in various fields, including Aerial photography, disaster relief, exploration, reconnaissance, agriculture[1].Compared to the traditional fixed-wing UAV, multi-rotor UAV does not need a runway for small space requirements. Compared to the traditional fixed-wing UAV, the ability of vertical takeoff and manipulating easily and hovering make multi-rotor UAV hasinherent advantages in the implementation of a variety of tasks. But multi-rotor UAV in weight and batterycapacity, there are many problems to be solved.

Control force of coaxial-rotor helicopter is provided by the same axis of rotation in the opposite direction of the two pairs of rotors, and the torque generated by the two rotors can cancel each other[2], so it does not require the tail rotor. Accordingly, coaxial rotor helicopter compared with single-rotor helicopter has many advantages. Inspired by coaxial-rotor helicopter, we combine coaxial propellers with multi-rotor copter, and employ coaxial propellers as rotorcraft main source of lift, the design structure diagram of the multi-rotor aircraft is shown in Figure I .

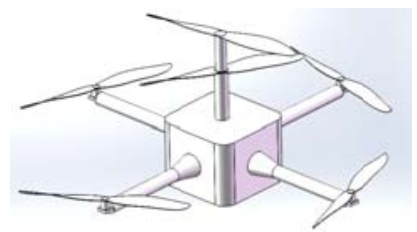

FIGURE I. DESIGN STRUCTURE DIAGRAM OF THE MULTI-ROTOR AIRCRAFT

In Figure I, speed of two coaxial propellers is controlled by the same motor through two sets of bevel gear, resulting in a total reaction torque to zero by the opposite direction of rotation in two coaxial propellers.

\section{DYNAMICS MODEL OF COAXIAL-ROTOR ROTORCRAFT}

\section{A. Selection of the Coordinate System}

To easily describe the attitude of the rotorcraft and simulation needs to define two coordinate systems: the first is the reference coordinate system OXYZ, select NED (North East ground) coordinate system as the reference coordinate system, the rotorcraft starting position as the origin of the coordinate position; the second coordinate system as the body coordinate system oxyz, fixedly connected with the rotorcraft, the origin of the coordinatecoincides with the position of the center of gravity position. The two coordinate systems are shown in Figure II.
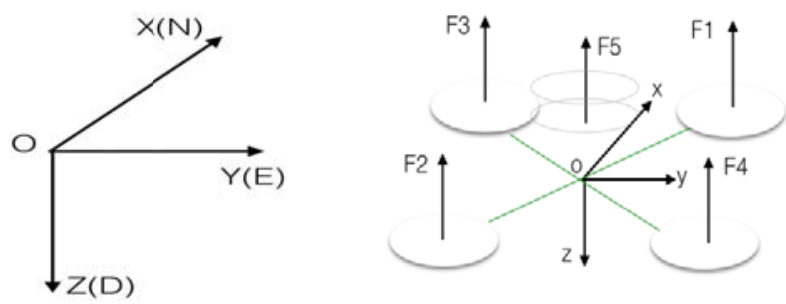

(a)Reference coordinate system(b)Body coordinate system FIGURE II. COORDINATE SYSTEM

To facilitate the conversion of two vectors in deferent coordinate systems, we need to define the Euler angles. First, the angle of rotation of rotorcraft around the z-axis in the body coordinate system is marked yaw angle $\varphi$, the angle of rotation of rotorcraft around the y-axis in the body coordinate system is marked pitch angle $\theta$, finally, the angle of rotation of rotorcraft around the $\mathrm{x}$-axis in the body coordinate system is marked roll angle $\phi$. Whereby we can acquire transformation matrix from the reference coordinate system to the body coordinate system, which is calulated as follows:

$$
\begin{aligned}
T_{E}^{B}(\phi, \theta, \varphi) & =R_{x}(\phi) R_{y}(\theta) R_{z}(\varphi) \\
= & {\left[\begin{array}{ccc}
C_{\theta} C_{\varphi} & C_{\theta} S_{\varphi} & -S_{\theta} \\
S_{\phi} S_{\theta} C_{\varphi}-C_{\phi} S_{\varphi} & S_{\phi} S_{\theta} S_{\varphi}+C_{\phi} C_{\varphi} & S_{\phi} C_{\theta} \\
C_{\phi} S_{\theta} C_{\varphi}+S_{\phi} S_{\varphi} & C_{\phi} S_{\theta} S_{\varphi}-S_{\phi} C_{\varphi} & C_{\phi} C_{\theta}
\end{array}\right] }
\end{aligned}
$$

In the above formula, $C_{\theta}=\cos \theta, S_{\phi}=\sin \phi$.

The use of more intuitive and easy direction cosine matrix, and can meet the needs of the simulation[3], so wo adopt the direction cosine matrix conversion between the two coordinate systems. 
In order to establish the dynamics model of the multi-rotor aircraft, we need to make the following assumptions:

a.gravity center of multi-rotor aircraft is in the origin of the body coordinate system and the center of gravity position unchanged.

b. motor shaft perpendicular to the plane of the body coordinate system xoy, unbiased and correctly installed. The motor of coaxial propellers spindle through the center of gravity.

c. multi-rotor aircraft is rigid body with constant quality.

d. during simulation process does not detect aerodynamic interference between coaxial propellers and other four rotors.

\section{B. Simplified Mechanical Model of the Multi-rotor Aircraft}

Simplified mechanical model of coaxial-rotor rotorcraft is shown in Figure III. Wherein $\mathrm{F}_{1} \sim \mathrm{F}_{4}$ are lift provided by the below four motors, $\mathrm{F}_{5}$ is generated by two coaxial propellers, $\mathrm{M}_{1} \sim \mathrm{M}_{4}$ are resistance moment subjected to shaft of the below four motors, $\mathrm{M}_{5}$ and $\mathrm{M}_{6}$ are resistance moment subjected to shaft of the two coaxial propellers, $\mathrm{G}$ is gravity of coaxial-rotor rotorcraft.

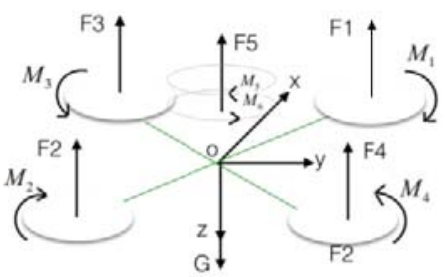

FIGURE III. SIMPLIFIED MECHANICAL MODEL OF COAXIAL-ROTOR ROTORCRAFT

Relationship between lift $\mathrm{F}$ generated by the below propellers and rotational speed $w$ is as follows[4]:

$$
F_{i}=k_{T} w^{2} \quad \mathrm{i}=(1 \sim 4)
$$

In the above formula, $k_{T}$ is lift coefficient of propellers.

Due to aerodynamic interference between coaxial propellers, lift $\mathrm{F}_{5}$ generated by two coaxial propellers can be acquired as follows:

$$
F_{5}=k_{e} k_{T} w^{2}
$$

Relationship between resistance moment $M$ and rotational speed $w$ is as follows:

$$
M_{i}=k_{D} w^{2} \quad \mathrm{i}=(1 \sim 4)
$$

In the above formula, $k_{D}$ is resistance coefficient of propellers.

By the classical mechanics, we can found any arbitrary motion of a free rigid body in space, it can be seen as just the center of mass translational motion and rotation around the center of mass motion synthesis [5], The basic equation is as follows:

$$
\begin{aligned}
& m \frac{d \vec{V}}{d t}=\vec{F} \\
& \frac{d \vec{H}}{d t}=\vec{M}
\end{aligned}
$$

In the above formula, $\vec{F}$ is main vector of external forces applied to coaxial-rotor rotorcraft, $\vec{M}$ is main moment of external forces applied to coaxial-rotor rotorcraft.

By analysis of the kinetic model of the multi-rotor aircraft, we can obtain dynamics scalar equations of centroid:

$$
\begin{aligned}
& m x=-\left(C_{\phi} S_{\theta} C_{\varphi}+S_{\phi} S_{\varphi}\right) \sum_{i=1}^{4} F_{i}-k_{x} x \\
& m y=-\left(C_{\phi} S_{\theta} S_{\varphi}-S_{\phi} C_{\varphi}\right) \sum_{i=1}^{4} F_{i}-k_{y} y \\
& m z=-C_{\phi} C_{\theta} \sum_{i=1}^{4} F_{i}+m g-k_{z} z
\end{aligned}
$$

In the above formula, $\mathrm{k}_{\mathrm{x}}, \mathrm{k}_{\mathrm{y}}$ and $\mathrm{k}_{\mathrm{z}}$ are coefficient of resistance along the axis of $\mathrm{x}, \mathrm{y}, \mathrm{z}$.

By the moment of momentum equation we can get kinetic scalar equations:

$$
\left[\begin{array}{l}
M_{x} \\
M_{y} \\
M_{z}
\end{array}\right]=\left[\begin{array}{c}
J_{x} \stackrel{\mathrm{g}}{\mathrm{p}}+\left(J_{z}-J_{y}\right) q r \\
J_{y} \stackrel{\mathrm{g}}{\mathrm{q}}+\left(J_{x}-J_{z}\right) \mathrm{pr} \\
J_{z} \stackrel{\mathrm{g}}{r}+\left(J_{y}-J_{x}\right) \mathrm{pq}
\end{array}\right]
$$

In the above formula, $J x, J y, J z$ are moment of inertial in the axis of $\mathrm{x}, \mathrm{y}, \mathrm{z} . M x, M y, M z$ are moment in the axis of $\mathrm{x}, \mathrm{y}$, $\mathrm{Z}$.

$\mathrm{U}_{1}$ is defined as the control amount of the rotorcraft in the vertical direction, $\mathrm{U}_{2}$ is defined as the roll control amount, $\mathrm{U}_{3}$ is defined as the pitch control amount, $\mathrm{U}_{4}$ is defined as the yaw control amount, the formula of the four control amount can be shown as follows:

$$
\left[\begin{array}{l}
U_{1} \\
U_{2} \\
U_{3} \\
U_{4}
\end{array}\right]=\left[\begin{array}{c}
F_{1}+F_{2}+F_{3}+F_{4}+F_{5} \\
F_{2}+F_{3}-\left(F_{1}+F_{4}\right) \\
F_{1}+F_{3}-\left(F_{2}+F_{4}\right) \\
M_{1}+M_{2}-\left(M_{3}+M_{4}\right)
\end{array}\right]=\left[\begin{array}{c}
\sum_{i=1}^{5} k_{t} w_{i}^{2} \\
k_{t}\left(w_{2}^{2}+w_{3}^{2}-w_{1}^{2}-w_{4}^{2}\right) \\
k_{t}\left(w_{1}^{2}+w_{3}^{2}-w_{2}{ }^{2}-w_{4}^{2}\right) \\
k_{d}\left(w_{1}{ }^{2}+w_{2}{ }^{2}-w_{3}{ }^{2}-w_{4}{ }^{2}\right)
\end{array}\right]
$$

To facilitate the research, we can describe the rotorcraft motion equations in the form of the state space, which is as follows:

$$
\begin{aligned}
& \stackrel{\mathrm{g}}{X}=A X+B U \\
& Y=C X+D U
\end{aligned}
$$


In the above formula, $X$ is the state vector, $U$ is input vector of the system, $Y$ is the output vector of the system, namely:

$$
\begin{aligned}
X & =\left[\begin{array}{lllllllll}
\phi & \theta & \varphi & \mathrm{g} & \mathrm{g} & \mathrm{g} & \mathrm{g} & \mathrm{g} & \mathrm{g} \\
\theta & \varphi & \mathrm{x} & \mathrm{y} & \mathrm{z} & \mathrm{g}
\end{array}\right]^{T} \\
U & =\left[\begin{array}{llllll}
U_{1} & U_{2} & U_{3} & U_{4}
\end{array}\right]^{T} \\
Y & =\left[\begin{array}{lllllll}
\mathrm{g} & \mathrm{g} & \mathrm{g} & \mathrm{g} & \mathrm{g} & \mathrm{g} \\
\phi & \dot{\theta} & \varphi & \mathrm{x} & y & \mathrm{z}
\end{array}\right]^{T}
\end{aligned}
$$

Taking into account the aircraft at low speed simulation, we make the following assumptions in order to linearizestate equations of coaxial-rotor rotorcraft:

a.ignore the cross impact of angular velocity,namely $\dot{\theta} \dot{\varphi}, \dot{\phi} \dot{\varphi}, \dot{\theta} \dot{\phi}$. $k_{\mathrm{z}}$

b.ignore rotorcraft coefficient of resistance,namely $k_{x}, k_{y}$,

After linearization we can get state equations of coaxial-rotor rotorcraft as follows:

$$
\stackrel{g}{X}=\left(\begin{array}{c}
\stackrel{g}{\phi} \\
g \\
\dot{g} \\
g \\
\varphi \\
\left(U_{2} l\right) / J_{x} \\
\left(U_{3} l\right) / J_{y} \\
\left(U_{4}\right) / J_{z} \\
-\left(C_{\phi} S_{\theta} C_{\varphi}+S_{\phi} S_{\varphi}\right) U_{1} / m \\
-\left(C_{\phi} S_{\theta} S_{\varphi}-S_{\phi} C_{\varphi}\right) U_{1} / m \\
-C_{\phi} C_{\theta} U_{1} / m+g \\
\end{array}\right)
$$

\section{CONTROL SYSTEM OF MULTI-ROTOR AIRCRAFT}

The rotorcraft in flight are more susceptible to outside interference, such as gust of wind or electromagnetic radiation. Thereby, we adopt double-loop PID control as the core control algorithms, with control of the angular as the outer loop and control of the angular velocity as the inner loop. The overall structure of the control system can be seen in Figure IV.

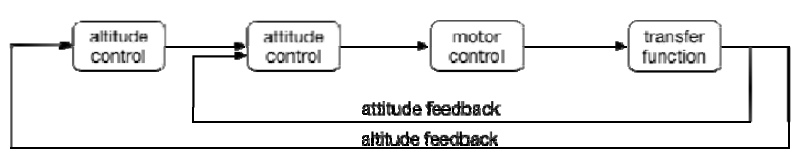

FIGURE IV. THE OVERALL STRUCTURE OF THE CONTROL SYSTEM

\begin{tabular}{|c|c|c|c|c|c|c|c|c|}
\hline $\begin{array}{l}\text { parame- } \\
\text { ters }\end{array}$ & 1 & $\mathrm{k}_{\mathrm{d}}$ & $\mathrm{k}_{\mathrm{t}}$ & $\mathrm{J}_{\mathrm{x}}$ & $\mathrm{J}_{\mathrm{y}}$ & $\mathrm{J}_{\mathrm{z}}$ & $\mathrm{m}$ & $\mathrm{k}_{\mathrm{e}}$ \\
\hline units & $\mathrm{m}$ & $\underset{s^{2}}{\mathrm{Nm}}$ & $\mathrm{Ns}^{2}$ & $\underset{2}{\mathrm{Nms}}$ & $\underset{2}{\mathrm{Nms}}$ & $\underset{2}{\mathrm{Nms}}$ & $\mathrm{Kg}$ & \\
\hline value & $\begin{array}{c}0.3 \\
5\end{array}$ & $\begin{array}{l}7.2 \mathrm{e} \\
-7\end{array}$ & $\begin{array}{c}2.85 \mathrm{e}- \\
4\end{array}$ & $\begin{array}{l}0.0 \\
21\end{array}$ & $\begin{array}{l}0.0 \\
23\end{array}$ & $\begin{array}{l}0.0 \\
41\end{array}$ & 4 & $\begin{array}{l}1 . \\
4\end{array}$ \\
\hline
\end{tabular}

Rotorcraft simulation parameters in this paper can be seen in Table I .

TABLE I. SIMULATION PARAMETERS

\section{A. Altitude Control Submodule}

Structure of altitude control submodule made by Simulink is shown in Figure $\mathrm{V}$.

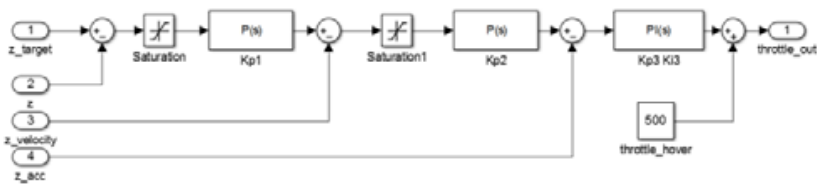

FIGURE V. STRUCTURE OF ALTITUDE CONTROL SUBMODULE

From Figure $\mathrm{V}$ we can see that input of altitude control submodule is altitude of target $\mathrm{z}_{\text {target}}$, with feedback of altitude, velocity and acceleration. Finally, pwm of motor caculated by two P controllers and one PI controller input to controller of posture.

\section{B. Attitude Control Submodule}

Structure of attitude control submodule made by Simulink can be seen in Figure VI.

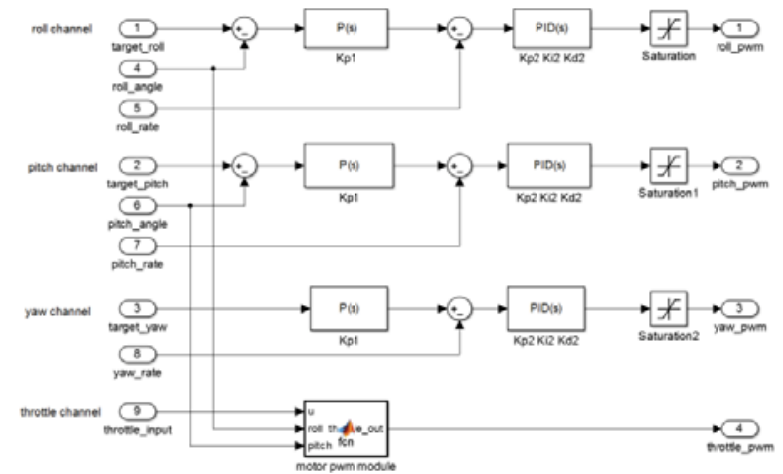

FIGURE VI. STRUCTURE OF ATTITUDE CONTROL SUBMODULE

From Figure VI, submodule of attitude control contains roll channel, pitch channel, yaw channel and throttle channel, wherein amount of throttle control obtained from submodule of altitude control output to submodule of motor control after addition with compensation value of roll angle and pitch angle. Input of roll channel is expected roll angle,input of pitch channel is expected pitch angle, input of yaw channel is expected yaw angular velocity. The controller of roll and pitch both have two loops:P loop for angular error and PID loop for angular rate error.

\section{Motor Control Submodule}

Structure of motor control submodule made by Simulink is shown in Figure VII.

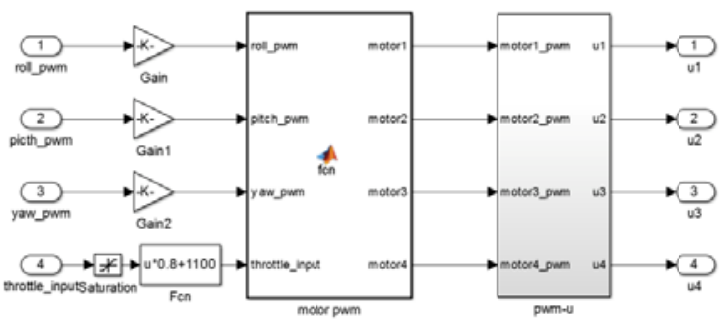

FIGURE VII. STRUCTURE OF MOTOR CONTROL SUBMODULE 
From Figure VII, input of motor control submodule is pwm value of roll, pitch, yaw and throttle obtained by submodule of posture control, then output of motor control submodule input to state space equation. Wherein relationship of the motor voltage and pwm value follows a linear model:

$$
V=22.2 / 1900 * p w m
$$

\section{Transfer Function Submodule}

Structure of transfer function submodule made by Simulink is shown in Figure VIII.

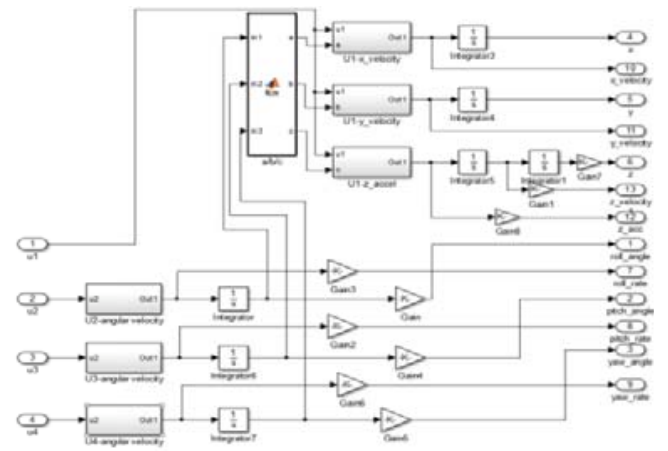

FIGURE VIII. STRUCTURE OF TRANSFER FUNCTION SUBMODULE

From Figure VIII, we can obtain output of system by the transfer function of $U$ and $Y$, from which we can acquire attitude angle and location information of the rotorcraft. So far, the control system of multi-rotor aircraft is complete.

\section{RESULTS OF SIMULATION}

We conduct simulation experiment through Simulink, PID parameters of altitude simulation and PID parameters of attitude simulation can be seen in Table II and Table III. Response curves of simulation are shown in Figure IX.

TABLE II. PID PARAMETERS OF ALTITUDE SIMULATION

\begin{tabular}{|c|c|c|c|}
\hline $\mathrm{K}_{\mathrm{P} 1}$ & $\mathrm{~K}_{\mathrm{P} 2}$ & $\mathrm{~K}_{\mathrm{P} 3}$ & $\mathrm{~K}_{\mathrm{I} 3}$ \\
\hline 1.1 & 4 & 2.3 & 2 \\
\hline
\end{tabular}

TABLE III. PID PARAMETERS OF ATTITUDE SIMULATION

\begin{tabular}{|c|c|c|c|c|}
\hline & $\mathrm{K}_{\mathrm{P} 1}$ & $\mathrm{~K}_{\mathrm{P} 2}$ & $\mathrm{~K}_{\mathrm{I} 2}$ & $\mathrm{~K}_{\mathrm{D} 2}$ \\
\hline roll & 4.5 & 0.5 & 0.2 & 0.004 \\
\hline pitch & 4.5 & 0.6 & 0.2 & 0.004 \\
\hline yaw & 1.1 & 0.2 & 0.1 & 0.01 \\
\hline
\end{tabular}
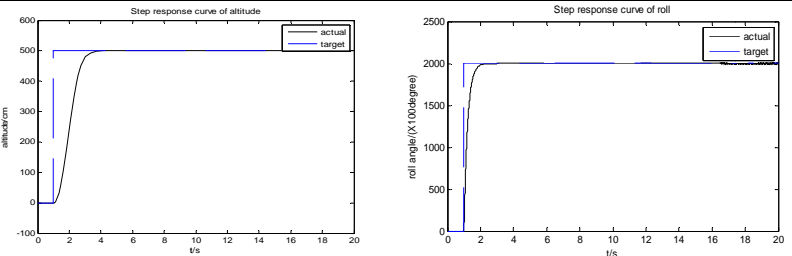
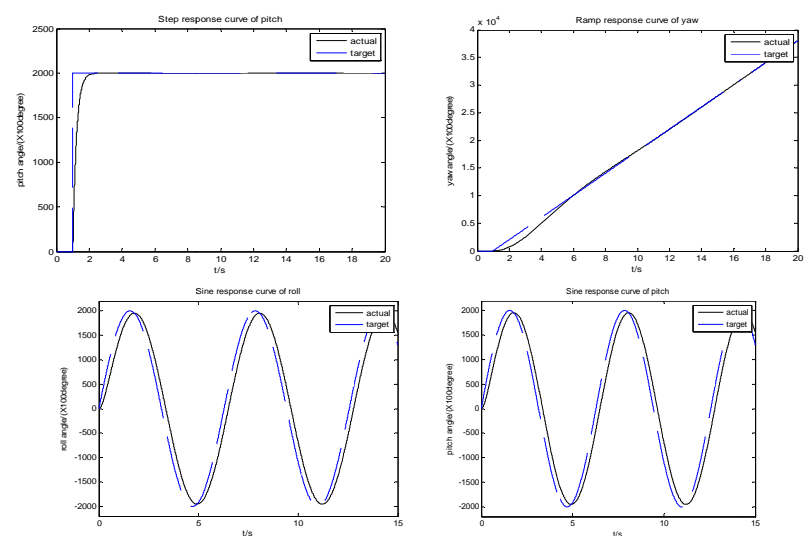

(a)Step response curve of altitude (b)Step response curve of roll (c)Step response curve of pitch (d)Ramp response curve of yaw (e)Sine response curve of roll (f)Sine response curve of pitch

FIGURE IX. RESPONSE CURVES OF SIMULATION

From Figure IX a, altitude of the control system controls is almost with no overshoot and responding fast, the steady-state error is almost zero. From Figure b and c, roll and pitch channels response fast, quickly reach the desired input value and no overshoot. Input of yaw channel is angular velocity, so target line is ramp function, and response of yaw channel is similar to a straight line and achieves the desired effect. From Figure e and f, channels of roll and pitch have a good follow-up performance and the lag is only about $0.2 \mathrm{~s}$.

\section{CONCLUSIONS}

In this paper, we presented a rotorcraft based on coaxial propellers, and it could enhance load capacity when compared with the conventional rotorcraft. Next, we designed the flight control system of multi-rotor aircraft based on the dynamics model. Finally, the simulation results show that the control system achieve effective control of attitude and altitude.

\section{ACKNOWLEDGMENTS}

This work is financially supported by NSAF (Grant N o. U1430113), and Key Laboratory of Dynamics and cont -rol of Flight Vehicle (Beijing Institute of Technology), M inistry of Education.

\section{REFERENCES}

[1] Feng Wang,Jiang Wu,Guo-Qing Zhou and Zheng-Hao Li, Development study of multi-rotor. Science \& Technology Vision,2015,(13):6-7.

[2] Ming Chen, Technology characteristic and development of coaxial rotor helicopter. Aeronautical Manufacturing Technology, 2009,(17):26-31.

[3] Mark E. Dreier. Introduction to helicopter and tiltrotor flight simulation.American Institute of Aeronautics and Astronautics, 2007, pp.24-25.

[4] Wei-Wei Qiao. Quadrotor aircraft flight control system and simulation. North University of China, 2012.

[5] Xing-Fang Qian,Rui-Xiong Lin and Ya-Nan Zhao. Mechanics of missile flight.Beijing Institute of Technology Press, 2012, pp. 89-91. 